\title{
O CONCEITO DE VULNERABILIDADE SOCIAL NO ÂMBITO DA PSICOLOGIA NO BRASIL: UMA REVISÃO SISTEMÁTICA DA LITERATURA
}

THE CONCEPT OF SOCIAL VULNERABILITY IN THE REALM OF PSYCHOLOGY IN BRAZIL: A SYSTEMATIC REVIEW OF THE LITERATURE

EL CONCEPTO DE VULNERABILIDAD SOCIAL EN EL ÁMBITO DE LA PSICOLOGÍA EN BRASIL: UNA REVISIÓN SISTEMÁTICA DE LA LITERATURA

\author{
Juliano Beck Scott \\ Caroline de Abreu Prola** \\ Aline Cardoso Siqueira ${ }^{* * *}$ \\ Caroline Rubin Rossato Pereira ${ }^{* * * *}$
}

\begin{abstract}
RESUMO
O conceito de vulnerabilidade social vem sendo utilizado em estudos de várias áreas, entre elas, a Psicologia, assumindo diversas conotações. Diante disso, este estudo teve como objetivo investigar o conceito de vulnerabilidade social empregado nas publicaçôes da área da Psicologia no Brasil. Para isso, realizou-se uma revisão sistemática da literatura nacional acerca do tema, obtendo-se 21 artigos publicados entre os anos de 2005 a 2013. Os resultados encontrados sugerem que a temática da vulnerabilidade social representa um objeto de pesquisa recente e de crescente interesse na produção científica nacional em Psicologia. Esses resultados permitem ainda concluir que existem diferentes definições para o conceito de vulnerabilidade social, já que não se encontrou uma única definição para o termo. No entanto essa temática tem aparecido em diversos estudos nos últimos anos, o que demonstra a sua relevância e importância no âmbito científico e social.
\end{abstract}

Palavras-chave: Vulnerabilidade social. Conceito. Psicologia.

\section{ABSTRACT}

The concept of social vulnerability has been used in studies approaching several different fields of knowledge, including Psychology, assuming a variety of senses. This study aims to investigate the concept of social vulnerability used in psychology-related publications in Brazil. In order

\footnotetext{
Texto recebido em 19 de março de 2015 e aprovado para publicação em 26 de abril de 2016.

*Doutorando em Psicologia pela Universidade Federal do Rio Grande do Norte (UFRN), mestre em Psicologia pela Universidade Federal de Santa Maria (UFSM), psicólogo.E-mail: bs.juliano@gmail.com.

** Mestranda em Psicologia pela Universidade Federal de Santa Maria (UFSM).

"** Doutora em Psicologia pela Universidade Federal de Santa Maria (UFSM).

${ }^{* * * *}$ Doutora em Psicologia pela Universidade Federal de Santa Maria (UFSM).
} 
to do that, a systematic review of the national literature on the subject was performed, resulting in 21 articles which were published from 2005 to 2013. The results suggest that social vulnerability has been the subject of recent researches and it has become an increasing interest in national scientific production in Psychology. The results also allowed the conclusion that there are different definitions of the concept of social vulnerability, whereas a single definition was not found. Nonetheless, this matter has been present in several studies in recent years, which shows its relevance and importance in the scientific and social contexts.

Keywords: Social vulnerability. Concept. Psychology.

\section{RESUMEN}

El concepto de vulnerabilidad social se ha utilizado en los estudios de varias áreas, entre ellas, la Psicología, asumiendo diversas connotaciones. Siendo así, este estudio tuvo como objetivo investigar el concepto de vulnerabilidad social empleado en las publicaciones en el área de la psicología en Brasil. Para ello, llevamos a cabo una revisión sistemática de la literatura nacional sobre el tema, lo que resultó en 21 artículos publicados entre los años 2005 a 2013. Los resultados sugieren que el tema de la vulnerabilidad social es un objeto de investigación reciente y de creciente interés en la producción científica nacional en Psicología. Los resultados permiten también concluir que existen diferentes definiciones del concepto de vulnerabilidad social, porque no encontramos una definición única del concepto. Sin embargo, este tema ha aparecido en varios estudios en los últimos años, lo que demuestra su relevancia e importancia en el contexto científico y social.

Palabras clave: Vulnerabilidad social. Concepto. Psicología.

\section{INTRODUÇÃO}

$\mathrm{D}$ esde a década de 1990, o conceito de vulnerabilidade e, de modo especial, vulnerabilidade social vem ganhando espaço na produção científica e no discurso daqueles que trabalham com saúde ou assistência social. Entretanto, conforme Figueiredo e Noronha (2008), ainda há poucos estudos e debates sobre o que realmente caracteriza a vulnerabilidade. Segundo os autores, a definição de vulnerabilidade vem sendo discutida mais atrelada ao termo minorias, por se entender que a população considerada vulnerável faz parte de um grupo de menor dominância social. Dessa forma, percebe-se que ser ou não vulnerável está associado à ideia de precariedade de condições de vida.

O conceito de vulnerabilidade surgiu na década de 1980, como resposta à epidemia de Human Immunodeficiency Virus (HIV)/Síndrome da Imunodeficiência Adquirida (aids), referindo-se às pessoas que apresentavam 
uma gama maior de fatores associados à ação patogênica do vírus. Percebe-se, assim, que esse conceito estava relacionado à saúde, resultante de um processo de interseções entre o ativismo suscitado pela epidemia do HIV/aids e o movimento dos direitos humanos. Isso fez com que a vulnerabilidade fosse inserida em discussões da saúde pública, ganhando maior notoriedade e espaço, o que a fez avançar para além do conceito epidemiológico de risco, grupo de risco e comportamento de risco (Ayres, França Júnior, Calazans \& Saletti Filho, 2009).

Ayres, França Júnior, Calazans \& Saletti Filho (2009) ressaltam que o conceito de vulnerabilidade se desenvolveu a partir da possibilidade de exposição da pessoa à infecção pelo vírus HIV e do possível adoecimento advindo da aids como resultado de aspectos individuais, coletivos e contextuais que ocasionam maior ou menor disposição de recursos voltados à proteção. Os autores também argumentam que o conceito de vulnerabilidade atenta para os diferentes contextos sociais como determinantes de variadas suscetibilidades. Isso faria com que as diferenças grupais e identitárias assumissem uma nova forma, compreendendo o todo social, ou seja, os segmentos populacionais mais expostos não seriam mais identificados por algum traço, mas sim pelo lugar ocupado ante o conjunto da sociedade. Portanto a epidemia do HIV/aids, por meio do termo vulnerabilidade, passou a ser vista como algo do social e não apenas de grupos particularizados como anteriormente. Isso demonstra uma evolução do termo e do seu entendimento na perspectiva da saúde.

O termo vulnerabilidade já assumiu várias conotações, entre estas, designando grupos ou indivíduos fragilizados, juridicamente ou politicamente, que necessitam de auxílio e proteção para a garantia de seus direitos como cidadãos. O vulnerável carrega, nesse sentido, a ideia do mais fraco, ou seja, aquele que está em desvantagem quanto ao critério de distribuição (renda, serviços, qualidade de vida, educação e saúde) e que é alvo de políticas públicas específicas de auxílio e de busca de garantia de direitos (Ayres et al., 2009; Figueiredo \& Noronha, 2008).

Ademais, vale salientar que o termo exclusão social antecede o conceito de vulnerabilidade social, tendo sido amplamente utilizado na definição de situações sociais como pobreza e marginalidade (Lopes, 2008). Diferentemente do que muitos pensam, o conceito de exclusão social, embora relacionado, não deve ser concebido como um sinônimo de pobreza. O termo pobreza faz parte de um fenômeno econômico integrante de uma relação direta entre capital e trabalho. Já a exclusão social, por sua vez, pode ser entendida como parte das relações sociais do contemporâneo, expressas pela precarização do trabalho, desqualificação social, desagregação identitária e desumanização do outro. Isso ocorre de uma forma que, "nos processos de exclusão produzidos no mundo neoliberal, mais que controlar ou negar o acesso ao trabalho ou ao consumo, a sociedade capitalista controla e nega a própria condição de sujeiticidade do 
indivíduo" (Lopes, 2008, p. 357). Portanto, conforme Castro e Abromavay (2002), com base numa lógica de mercado e de capital social, vulneráveis seriam aqueles que se encontram fora do sistema.

O termo exclusão social foi ainda utilizado por um longo tempo, sendo posteriormente substituído pelo conceito de "vulnerabilidade social". A vulnerabilidade social foi gradativamente sendo incorporada às questóes sociais. Inicialmente, esse conceito estava relacionado às políticas sociais na maioria dos países e ao papel por elas desempenhado. Conforme a concepção foi se consolidando, novos estudos foram sendo realizados, trazendo diferentes enfoques (Brasil, 2007).

Pedersen e Silva (2013) afirmam que atualmente o conceito de vulnerabilidade social tem sido usado para caracterizar uma parcela da população, cada vez maior, que se encontra em uma situação desfavorável em relação a outros grupos populacionais. Essa conceituação surge, principalmente, a partir do fim do século XX e início do século XXI devido às modificações trazidas pelo mercado de trabalho. Dessa forma, aqui são abordados tanto os desempregados como aqueles que se encontram em situações desfavoráveis de emprego e geração de renda.

De acordo com Morais, Raffaelli e Koller (2012), o conceito de vulnerabilidade social pode ser aplicado a pessoas que vivenciam situações de adversidade em seu cotidiano, ou seja, a vulnerabilidade social pode estar associada a fatores de risco que afetam negativamente as pessoas e seu cotidiano. São considerados fatores de risco aqueles cujas condições ou variáveis provocam efeitos negativos ou indesejáveis, ou até mesmo comportamentos que comprometam a saúde e o bem-estar do indivíduo. Entre os fatores de risco, estão os comportamentos de risco que podem ser igualmente prejudiciais. Para Paulino e Lopes (2010), comportamento de risco pode ser definido como açóes ou atividades que aumentam a probabilidade de consequências adversas para o desenvolvimento psicossocial. Essas consequências, por sua vez, podem desencadear ou, até mesmo, agravar danos ou doenças para quem as realizou. Dessa forma, entendese que, no comportamento de risco, existem variáveis psicológicas e sociais que interferem no bem-estar do indivíduo (Koller, De Antoni \& Carpena, 2012; Morais, Morais, Reis \& Koller, 2010).

O conceito de vulnerabilidade social pode aparecer sob um outro formato que, conforme a Política Nacional de Assistência Social (Brasil, 2004), não está, necessariamente, ligado à pobreza ou a questões econômicas. As próprias características do Estado interferem nessa definição, pois as desigualdades características de uma dada estrutura social interferem no grau de vulnerabilidade dos grupos a ela pertencentes. Isso faria com que a vulnerabilidade surgisse de um resultado negativo entre a "disponibilidade de recursos materiais e simbólicos dos 
atores, sejam eles indivíduos ou grupos, e o acesso à estrutura de oportunidades sociais, econômicas, culturais que preveem do Estado, do mercado e da sociedade" (Figueiredo \& Noronha, 2008, p. 131). Isso explicaria muitas das dificuldades enfrentadas por uma grande parcela da população brasileira quanto ao desempenho e à mobilidade social. Entende-se aqui desempenho e mobilidade social como a dificuldade encontrada por determinadas parcelas populacionais em ascender de posição social ou até mesmo melhorar sua qualidade de vida frente às dificuldades enfrentadas.

A partir do exposto, percebe-se não haver uma definição unívoca ou simples do conceito de vulnerabilidade social. No âmbito da Psicologia, conforme essa área se aproxima de problemáticas relativas à saúde pública, à assistência social e às políticas públicas, torna-se fundamental que os profissionais tenham clareza a respeito de conceitos como o de vulnerabilidade social. Dessa forma, visando a um melhor entendimento do uso desse conceito, assim como de seus significados, este estudo tem como objetivo investigar a concepção de vulnerabilidade social empregada nas publicações nacionais da área da Psicologia.

\section{MÉTODO}

Este estudo é uma revisão sistemática da literatura que, conforme Rother (2007), refere-se a um método de pesquisa que parte de publicaçoos científicas em meios eletrônicos, com o objetivo de auxiliar na construção de um novo estudo. A revisão sistemática

é uma revisão planejada para responder uma pergunta específica e que utiliza métodos explícitos e sistemáticos para identificar, selecionar e avaliar criticamente os estudos, e para coletar e analisar os dados destes estudos incluídos na revisão (Castro, 2001, p. 5).

Nesse sentido, para este estudo, os critérios de inclusão para seleção dos artigos foram: ter como palavra-chave "vulnerabilidade social"; ser redigido por autores brasileiros da Psicologia; estar escrito em português, contemplando, dessa forma, a produção referente a esse conceito na área da Psicologia no Brasil; e ter sido produzido no período de 2003 a 2013.

Para a análise, desenvolveram-se quatro passos:

a) busca de evidências;

b) definição dos critérios de inclusão;

c) revisão e seleção dos estudos; e

d) apresentação dos resultados. 
Assim, realizou-se a busca de artigos completos no portal Periódicos CAPES, no ano de 2014, com base no descritor "vulnerabilidade social", sendo encontrados 308 artigos indexados, sendo desses 176 em idioma português. Foram, a partir disso, excluídas as produções de Portugal e os artigos que abordavam o tema da vulnerabilidade pelo enfoque epidemiológico, de desastres naturais e de áreas que não a Psicologia, como Nutrição, Enfermagem e Geografia, resultando em 21 artigos, que foram submetidos à análise.

O estudo tem caráter misto, pois emprega o método quantitativo e qualitativo, de forma combinada. De acordo com Sampieri, Collado e Lúcio (2006), esses dois enfoques contribuem com os estudos científicos, pois são complementares e possibilitam o benefício de ambos os métodos. Assim, o método quantitativo destacou-se na análise dos dados referentes ao ano de publicação e metodologia. Quanto ao método qualitativo, a análise de conteúdo (Bardin, 2004) foi empregada na organização e classificação dos conceitos de vulnerabilidade social contidos nos artigos selecionados. Com base nessa análise, os conceitos encontrados foram organizados em três categorias constituídas a posteriori, visando a um melhor entendimento dos significados atribuídos ao conceito de vulnerabilidade social nos artigos.

\section{RESULTADOS E DISCUSSÃO}

Os resultados apontam que a produção nacional, no âmbito da Psicologia, considerando o período apresentado de dez anos (2003 a 2013), mencionou o termo vulnerabilidade social somente no intervalo de 2005 a 2013, conforme a figura 1.

Figura 1. Produção nacional que utiliza o termo vulnerabilidade social

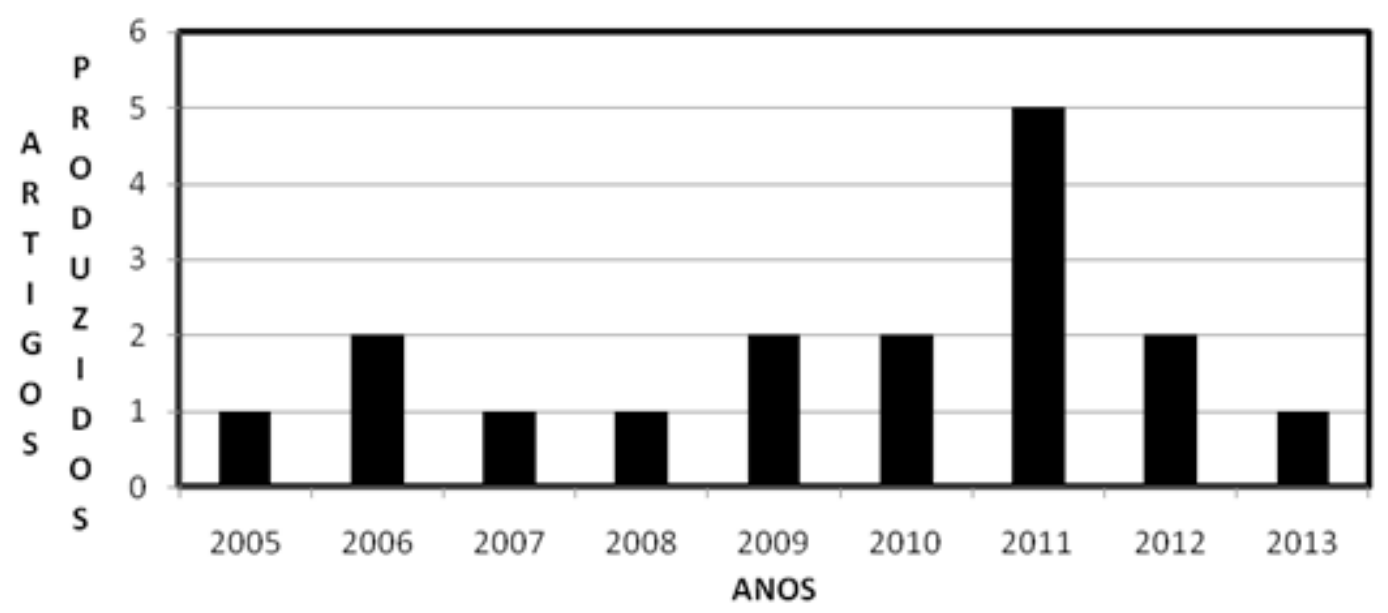

Fonte: Elaborado pelo autor. 
Esses resultados indicam que as produções acerca do tema tiveram um aumento significativo no ano de 2011, demonstrando um declínio nos dois anos seguintes, comparável aos dois primeiros anos apresentados, 2005 e 2006, o que demonstra um descompasso quanto ao uso do termo vulnerabilidade social nas produções em Psicologia no Brasil.

No que tange ao método, entre os 21 artigos analisados, 15 referiam-se a estudos empíricos e 6 a estudos teóricos. Dentre os artigos empíricos, apenas um empregou método de pesquisa de caráter quantitativo, sendo os demais de cunho qualitativo.

A esse respeito, considerando-se a vulnerabilidade social como um fenômeno multifatorial, compreende-se o predomínio de artigos com método qualitativo a partir da necessidade de um olhar que contemple as subjetividades e as entrelinhas do que está sendo investigado. Segundo Minayo (2010), a pesquisa qualitativa visa a apreender o objeto de estudo pelos significados, a realidade subjetiva e a compreensão do fenômeno e não pela quantificação de uma realidade.

Além disso, destaca-se que a pesquisa qualitativa pode ser considerada uma estratégia importante de investigação em um campo sobre o qual ainda não há um conhecimento consolidado. Uma vez que a utilização desse recurso metodológico possibilita o acesso ao significado atribuído pelos sujeitos aos fatos, relações, práticas e fenômenos sociais, torna-se possível a construção de um conceito que perpassa pela subjetividade e impressões (Lucchese \& Barros, 2007).

Com relação à população investigada nos estudos empíricos, destaca-se que grande parte das pesquisas teve como participantes profissionais de saúde e adolescentes. Entre os 15 estudos empíricos, 46,5\% tiveram profissionais de saúde como participantes; $38,7 \%$, adolescentes; e $14,8 \%$, outros participantes (usuários de serviços públicos, mulheres, crianças e profissionais da educação).

Pode-se pensar que o predomínio dos profissionais de saúde como fonte de informação para os estudos acerca da vulnerabilidade social deve-se à sua inserção e o seu contato diário e constante com serviços situados em regiōes consideradas vulneráveis em diferentes municípios. Assim, esses profissionais foram considerados pelos pesquisadores como ricos informantes acerca de suas vivências e auxiliares na construção do conhecimento sobre as populações pesquisadas.

Ainda em relação aos participantes, um número significativo de estudos empíricos voltou-se à população adolescente. Nessa fase do desenvolvimento, o sujeito necessita definir o seu papel dentro do círculo social no qual está inserido. 
Novas relações interpessoais são vivenciadas e estabelecidas, sendo o grupo de iguais um dos espaços mais importantes para a consolidação da identidade adolescente (Brêtas, Moreno, Eugenio, Sala, Vieira, Bruno, 2008; Outeiral, 2003). Logo, no caminho até a consolidação de uma identidade adulta, o adolescente pode se tornar vulnerável a determinadas situações de risco individual ou social, uma vez que se expõe a experiências para consolidar aspectos da sua personalidade.

A fim de analisar o conceito de vulnerabilidade social empregado nos estudos, realizou-se uma análise qualitativa do conteúdo presente nos artigos. Os dados encontrados foram divididos em três categorias temáticas: vulnerabilidade social como exposição a riscos; vulnerabilidade social a partir de aspectos demográficos e, ou, socioeconômicos; vulnerabilidade social a partir de uma noção multidimensional.

\subsection{Vulnerabilidade social como exposição a riscos}

Dos 21 artigos analisados, 5 utilizaram o termo vulnerabilidade social relacionado à exposição a riscos (Areco, Matias, Silva \& Simon, 2011; Koerich, Baggio, Backes, Backes, Carvalho, Meirelles, Erdmann, 2010; Navarro, Bezerra, Oliveira, Moreira, Alves, Gurgel, 2011; Sousa, Espírito Santo \& Motta, 2008; Tarachuque \& Souza, 2013). Essa relação remonta a uma perspectiva de vulnerabilidade social associada a situações de grande exposição a riscos, como doenças infectocontagiosas e situações desfavoráveis, tanto sociais como políticas, que podem afetar o bem-estar do indivíduo, da família ou grupo. Conforme segue:

A vulnerabilidade social ao HIV/Aids, vivenciada pelas mulheres, poderia [...] ser explicada por meio da inter-relação de fatores de diferentes naturezas, que compreenderiam três dimensões: a individual, decorrente em última instância da dificuldade de acesso às informaçōes acerca da prevenção e dos meios para concretizá-las, como o preservativo e uso de informes educativos; a social, resultante do acesso a serviços públicos, como educação e saúde, aptos a reduzir a vulnerabilidade individual; e, por último, a política, determinada pela elaboração e implementação das políticas de saúde para combate ao HIV/Aids (Sousa et al., 2008, p. 104).

Diante disso, segundo Ayres et al. (2009), o conceito de vulnerabilidade social, ligado a doenças infectocontagiosas, como o HIV/aids, surgiu da exposição de alguns indivíduos à infecção pelo vírus e do adoecimento causado pelo desenvolvimento da doença. Os diferentes aspectos contextuais, individuais ou coletivos, influenciariam na determinação dos grupos de risco à infecção, ou seja, da população que estaria mais vulnerável socialmente à infecção. 
Nessa perspectiva, de acordo com o Ministério do Trabalho e Emprego (Brasil, 2004), as situaçôes de vulnerabilidade social devem ser analisadas com base na capacidade de os indivíduos lidarem e enfrentarem situações de risco. Portanto a vulnerabilidade social estaria relacionada à aptidão de indivíduos, famílias ou grupos para controlarem as forças que afetam negativamente seu bem-estar, ou seja, se têm recurso para enfrentar as dificuldades e manter o equilíbrio, assim como também recursos para lidar com as adversidades.

Os artigos selecionados demonstraram aspectos sociais e políticos envolvidos na exposição a riscos:

Enquanto a noção de grupos de risco tende a individualizar e personificar a adversidade vivida, relacionando-a a uma questão de conduta, a perspectiva de vulnerabilidade social propóe-se a entendê-la como resultado de um processo social que remete à condição de vida e aos suportes sociais (Morais et al., 2012, p. 119).

Em um dos artigos, foi considerado como vulnerável aquele grupo de pessoas que estava exposto aos riscos oriundos das drogas, tráfico e violência, relacionando tal situação à conduta dos sujeitos. Isso ratifica a ideia de que "a noção de grupos de risco tende a individualizar e personificar a adversidade vivida (seja o uso de droga, a AIDS, etc.)" (Morais, Raffaelli \& Koller, 2010, p. 788). Nesse excerto, fica claro que a conduta do indivíduo também pode predispor a situaçôes de vulnerabilidade e risco.

\subsection{Vulnerabilidade social baseada em aspectos demográficos e, ou, socioeconômicos}

Essa categoria designa a definição de vulnerabilidade social com base nos aspectos demográficos e socioeconômicos (Sant'Anna, Aerts \& Lopes, 2005), as condições de saúde e a privação e, ou, dificuldade de acesso a direitos sociais básicos (Mandú, Antiqueira \& Lanza, 2008). Nesta revisão, foram encontrados 10 artigos (Calazans, Cappellini, Sequeira, Vieira, França Junior, 2006; Dellazzana \& Freitas, 2010; Farias \& Moré, 2011; Jucá et al., 2007; Moura, Lefevre \& Moura, 2012; Oliveira-Monteiro, 2010; Sant'Anna et al., 2005; Silva, Rozenberg, Bonan, Chuva, Costa, Gomes, 2011; Souza, Dutra-Thomé, Schiró, Morais, Koller, 2011; Zaniol, Dal Molin \& Andreoli, 2007) que empregaram essa definição, o número mais expressivo dessa análise. Segundo Areco, Matias, Silva, e Simon (2011), essa definição contempla, ainda, a relação do termo vulnerabilidade social com os benefícios dos programas sociais. Esses aspectos podem resultar em desigualdades sociais, processos de exclusão e, ou, manutenção de violência nas relações. Portanto esta perspectiva defende que a vulnerabilidade social surge quando os recursos materiais e simbólicos não são compatíveis, gerando, por consequência, um resultado negativo: 
Por vulnerabilidade social entende-se o resultado negativo da relação entre disponibilidade dos recursos materiais ou simbólicos dos atores, sejam eles indivíduos ou grupos, e o acesso à estrutura de oportunidades sociais, econômicas e culturais oriundas do Estado, do mercado e da sociedade (Morais, Raffaelli \& Koller, 2012, p. 119).

Essa relação adviria de uma insuficiência de oportunidades no contexto em que o indivíduo está inserido e de sua dificuldade em lidar com isso. Essa situação tornaria as oportunidades oferecidas pelo Estado e pela sociedade mais distantes e inalcançáveis para uma determinada população, interferindo negativamente em seu desenvolvimento.

\subsection{Vulnerabilidade social a partir de uma noção multidimensional}

Essa categoria, contemplada em seis artigos analisados (Areco et al., 2011; Bellenzani \& Malfitano, 2006; Macedo \& Kublikowski, 2009; Morais et al., 2010; Morais et al., 2012; Muramoto \& Mângia, 2011), abrange um olhar mais complexo sobre a conceituação de vulnerabilidade social, considerando outras variáveis. Conforme um dos artigos analisados:

A vulnerabilidade social das pessoas, famílias ou comunidades é então entendida como uma conjugação de fatores que pode afetar o nível de bem-estar das pessoas, famílias ou comunidades e que resulta em uma exposição maior ao risco. Trata-se, assim, de uma noção multidimensional, pois a vulnerabilidade pode afetar pessoas, grupos e comunidades em diferentes planos de seu bem-estar, de formas diferentes e em diferentes intensidades, estando relacionada à capacidade dos envolvidos de controlar os recursos requeridos para o aproveitamento de oportunidades propiciadas pelo Estado, pelo mercado e pela sociedade (Macedo \& Kublikowski, 2009, p. 692).

Conforme Pedersen e Silva (2013), a vulnerabilidade social extrapola a dimensão material, ou seja, a falta de recursos financeiros, devendo ser analisada com base na inter-relação entre os diversos fatores que compóem a estrutura social. Isso quer dizer que a vulnerabilidade social perpassa desde a inserção do sujeito no mercado de trabalho, a qualidade de suas relações sociais, os serviços a que tem acesso ou dispõe e as formas de proteção proporcionadas pelo Estado e que interferem na sua qualidade de vida e bem-estar.

Nesse sentido, a vulnerabilidade social pode ser entendida como:

A consideração de aspectos como baixa escolarização, relações familiares violentas ou conflituosas, ausência ou insuficiência de recursos financeiros e serviços de saúde, além de poucas perspectivas profissionais e de futuro, constituem-se, portanto, enquanto fatores individuais, coletivos e contextuais que configuram o que se chama de vulnerabilidade social (Farias \& Moré, 2011, p. 597). 
Segundo Bellenzani e Malfitano (2006), ainda é possível abordar o conceito desdobrando-o em duas dimensões: vulnerabilidade social e vulnerabilidade psíquica.

O conceito de vulnerabilidade psíquica, que propomos, nos parece pertinente pela possibilidade de pensarmos fatores potenciais de modo que, sinergicamente, componham condiçōes propulsoras ao sofrimento ou ao adoecimento psíquico. Esses fatores estariam relacionados tanto ao universo cultural, histórico e social, daí a dimensão da vulnerabilidade social, como às experiências de vida singulares que, combinados, seriam a matéria-prima para a constituição das subjetividades (Bellenzani \& Malfitano, 2006, p. 122).

Dessa forma, ao discutir o conceito de vulnerabilidade social, "pretendese sair de análises de posições, morfologias estáticas, e reconhecer processos contemporâneos, remodelações de relações sociais nas quais, sublinhamos, a cultura e a subjetividade" (Castro \& Abramovay, 2002, p. 145).

\section{CONSIDERAÇŌES FINAIS}

Com base neste estudo, percebe-se que a produção científica acerca da vulnerabilidade social no cenário brasileiro configura-se como uma temática ainda recente e pouco explorada na literatura da área da Psicologia. Foram encontrados, em sua maioria, estudos empíricos e qualitativos que visam a abarcar a complexidade envolvida nessa concepção. Identificou-se um descompasso na produção científica nacional sobre o conceito de vulnerabilidade social, demonstrando que esse tema ainda é um objeto de pesquisa pouco explorado na Psicologia.

Com base nos resultados, pode-se inferir que houve diferentes definições para o conceito de vulnerabilidade social. Nos últimos anos, essa temática tem aparecido em diversos estudos, entretanto sem definição única. Inicialmente, a vulnerabilidade foi associada a aspectos epidemiológicos e individualizantes, com o foco da vulnerabilidade social no sujeito, principalmente naqueles casos relacionados ao HIV/aids. Posteriormente, o conceito foi atrelado a aspectos demográficos e socioeconômicos da população, e essa definição abordou as condições de saúde, o acesso aos serviços básicos, a privação de direitos e a escassez de recursos materiais. Nessa concepção, a vulnerabilidade social é vista como desequilíbrio entre recursos materiais e simbólicos disponíveis ao sujeito e suas necessidades.

Em seguida, o conceito passou a levar em consideração uma multiplicidade de fatores imbricados na perspectiva da vulnerabilidade social. Entre os diversos fatores, estão as condiçôes socioeconômicas, os acessos aos serviços, a cultura prevalente, as relaçóes sociais e a própria subjetividade. 
A partir do momento em que ocorre a união desses vários aspectos, constituise a vulnerabilidade social, principalmente pela forma como o sujeito se relaciona com esses diversos fatores. Essa construção multidimensional vem ao encontro dos estudos preconizados pela Psicologia, que visam a oferecer um olhar de maior amplitude sobre o sujeito, percebendo os vários fatores implicados em sua subjetividade. Nesse sentido, a Psicologia como ciência possibilita a compreensão da subjetividade presente nas interações entre os indivíduos e entre o indivíduo e o social, considerando a influência dos comportamentos dos sujeitos sobre os grupos. Portanto uma reflexão acerca do conceito de vulnerabilidade social pode auxiliar outras áreas que empregam essa temática em seus estudos e atuação profissional.

A revisão sistemática do conceito de vulnerabilidade social abordou um período cronológico da produção científica e, portanto, entende-se que este estudo apresenta limitaçóes, tendo em vista que se realizou um recorte da produção científica conforme os critérios utilizados pelos pesquisadores. Entretanto este estudo serve como ponto de partida para futuras reflexóes, tendo em vista a evolução desse conceito, que aparece interligado com as relações e contextos sociais, podendo auxiliar os profissionais envolvidos com a temática na sua atuação profissional e inserção social. 


\section{REFERÊNCIAS}

Areco, N. M., Matias, C. A., Silva, R. C. \& Simon, C. P. (2011). Caracterização dos serviços que atendem adolescentes: interfaces entre saúde mental e drogadição. Psicologia \& Sociedade, 23(1), 103-113.

Ayres, J. R., França Júnior, I., Calazans, G. J. \& Saletti Filho, H. C. (2009). O conceito de vulnerabilidade e as práticas de saúde: novas perspectivas e desafios. In D. Czeresnia (Org.). Promoção da saúde: conceitos, reflexões, tendências. (2a ed.), Rio de Janeiro: Fiocruz.

Bardin, L. (2004). Análise de conteúdo. (4a ed.). Lisboa: Edições 70.

Bellenzani, R. \& Malfitano, A. P. S. (2006). Juventude, vulnerabilidade social e exploração sexual: um olhar a partir da articulação entre saúde e direitos humanos. Saúde e Sociedade, 15(3), 115-130.

Brasil. Ministério do Desenvolvimento Social e Combate à Fome. Conselho Nacional de Assistência Social. (2004). Política Nacional de Assistência Social. Brasília: Ministério do Desenvolvimento Social e Combate à Fome.

Brasil. Ministério do Trabalho e Emprego (2007). Aspectos conceituais da vulnerabilidade social. Brasília: MTE.

Brêtas, J. R. S., Moreno, R. S., Eugenio, D. S., Sala, D. C. P., Vieira, T. F. $\&$ Bruno, P. R. (2008). Os rituais de passagem segundo adolescentes. Acta Paulista de Enfermagem, 21(3), 404-411.

Calazans, G., Kiss, L., Cappellini, S., Sequeira, D., Vieira, M. R. \& França Junior, I. (2006). Plantôes jovens: acolhimento e cuidado por meio da educação entre pares para adolescente e jovens nos Centros de Testagem e Aconselhamento CTA. Saúde e Sociedade, 15(1), 22-36.

Castro, A. A. (2001). Revisão sistemática e meta-análise. Compacta: Temas de Cardiologia, 3(1), 5-9.

Castro, M. G. \& Abramovay, M. (2002). Jovens em situação de pobreza, vulnerabilidades sociais e violências. Cadernos de Pesquisa, 116, 143-173.

Dellazzana, L. L. \& Freitas, L. B. L. (2010). Um dia na vida de irmãos que cuidam de irmãos. Psicologia: Teoria e Pesquisa, 26(4), 595-603. 
Farias, R. \& Moré, C. O. O. (2011). Repercussões da gravidez em adolescentes de 10 a 14 anos em contexto de vulnerabilidade social. Psicologia: Reflexão e Critica, 25(3), 596-604.

Figueiredo, I. \& Noronha, R. L. (2008). A vulnerabilidade como impeditiva/ restritiva do desfrute de direitos. Revista de Direitos e Garantias Fundamentais, $4,129-146$.

Jucá, V. J. S., Silva, A. C. N., Passos, C. M., Castro, G. A., Melo, G. B., Tortorella, I., . . Reis, S. (2007). Significando a morte através de redes sociais em um contexto de vulnerabilidade social: um estudo com crianças pré-escolares e seus professores. Psicologia \& Sociedade, 19(2), 122-130.

Koerich, M. S., Baggio, M. A., Backes, M. T. S., Backes, D. S., Carvalho, J. N., Meirelles, B. H. S. \& Erdmann, A. L. (2010). Sexualidade, doenças sexualmente transmissíveis e contracepção: atuação da enfermagem com jovens de periferia. Revista Enfermagem UERJ, 18(2), 265-271.

Koller, S. H., De Antoni, C. \& Carpena, M. E. F. (2012). Família de crianças em situação de vulnerabilidade social. In M. N. Baptista \& M. L. M. Teodoro (Orgs.), Psicologia de família: teoria, avaliação e intervenção. (pp. 156-165). Porto Alegre: Artmed.

Lopes, J. R. (2008). Processos sociais de exclusão e políticas públicas de enfrentamento da pobreza. Caderno CRH, 21(53), 349-363.

Lucchese, R. \& Barros, S. (2007). A utilização do grupo operativo como método de coleta de dados em pesquisa qualitativa. Revista Eletrônica de Enfermagem, 9 (3), 798-805.

Macedo, R. M. S. \& Kublikowski, I. (2009). Valores positivos e desenvolvimento adolescente: perfil de jovens paulistanos. Psicologia em Estudo, 14, 689-698.

Mandú, E. N. T., Antiqueira, V. M. A. \& Lanza, R. A. C. (2009). Mortalidade materna: implicações para o programa saúde da família. Revista Enfermagem UERJ, 17(2), 278-284.

Minayo, M. C. (2010). Pesquisa social: teoria, método e criatividade. (29a ed.). Petrópolis: Vozes.

Morais, N. A., Koller, S. H. \& Raffaelli, M. (2010). Eventos estressores e indicadores de ajustamento entre adolescentes em situação de vulnerabilidade social no Brasil. Univ. Psychol., 9(3), 787-806. 
Morais, N. A., Morais, C. A., Reis, S. \& Koller, S. H. (2010). Promoção de saúde e adolescência: um exemplo de intervenção com adolescentes em situação de rua. Psicologia \& Sociedade, 22(3), 507-518.

Morais, N. A., Raffaelli, M. \& Koller, S. H. (2012). Adolescentes em situação de vulnerabilidade social e o continuum risco-proteção. Avances en Psicología Latinoamericana, 30(1), 118-136.

Moura, L. B. A., Lefreve, F. \& Moura, V. (2012). Narrativas de violências praticadas por parceiros íntimos contra mulheres. Ciência \& Saúde Coletiva, $17(4), 1025-1035$.

Muramoto, M. T. \& Mângia, E. F. (2011). A sustentabilidade da vida cotidiana: um estudo das redes sociais de usuários de serviço de saúde mental no município de Santo André (SP, Brasil). Ciência \& Saúde Coletiva, 16(4), 2165-2177.

Navarro, A. M. A., Bezerra, V. P., Oliveira, D. A., Moreira, M. A. S. P., Alves, M. S. C. F. \& Gurgel, S. N. (2011). Representaçôes sociais do HIV/AIDS: percepções dos profissionais da atenção primária à saúde. Revista de Pesquisa: Cuidado é Fundamental, 3(5, esp.), 92-99.

Oliveira-Monteiro, N. R. (2010). Percursos da gravidez na adolescência: estudo longitudinal após uma década de gestação. Psicologia: Reflexão e Crítica, 23(2), 278-288.

Outeiral, J. (2003). Adolescer: estudos revisados sobre adolescência. Rio de Janeiro: Revinter.

Paulino, J. A.\& Lopes, R. F. F. (2010). Relação entre percepção e comportamento de risco e níveis de habilidades cognitivas em um grupo de adolescentes em situação de vulnerabilidade social. Psicologia Ciência e Profissão, 30(4), 752765 .

Pedersen, J. R. \& Silva, J. A. (2013). A exploração sexual de crianças e adolescentes e sua relação com a vulnerabilidade social das famílias: desafios à garantia de direitos. In K. B. Krüger \& C. F. Oliveira. (Orgs.), Violência intrafamiliar: discutindo facetas e possibilidades. (pp. 45-64). Jundiaí: Paco.

Rother, E. T. (2007). Editorial: revisão sistemática x revisão narrativa. Acta Paulista de Enfermagem, 20(2), V-VI.

Sampieri, R. H., Collado, C. F. \& Lucio, P. B. (2006). Metodologia de pesquisa (3a ed.). F. C. Murad, M. Kassner \& S. C. D. Ladeira (Trad.). São Paulo: McGraw-Hill. 
Sant'Anna, A., Aerts, D. \& Lopes, M. J. (2005). Homicídios entre adolescentes no sul do Brasil: situaçôes de vulnerabilidade segundo seus familiares. Caderno de Saúde Pública, 21(1), 120-129.

Silva, K. S., Rozenberg, R., Bonan, C., Chuva, V. C. C., Costa, S. F. \& Gomes, M. A. S. M. (2011). Gravidez recorrente na adolescência e vulnerabilidade social no Rio de Janeiro (RJ, Brasil): uma análise de dados do Sistema de Nascidos Vivos. Ciência \& Saúde Coletiva, 16(5), 2485-2493.

Souza, A. P. L., Dutra-Thomé, L., Schiró, E. D. B. D., Morais, C. A. \& Koller, S. H. (2011). Criando contextos ecológicos de desenvolvimento de direitos humanospara adolescentes. Paidéia, 21(49), 273-278.

Sousa, M. C. P., Espírito Santo, A. C. G. \& Motta, S. K. A. (2008). Gênero, vulnerabilidade das mulheres ao HIV/Aids e ações de prevenção em bairro da periferia de Teresina, Piauí, Brasil. Saúde e Sociedade. São Paulo, 17(2), 58-68.

Tarachuque, J. \& Souza, W. (2013). Bioética e vulnerabilidade da população em situação de rua: um estudo a partir da realidade de Curitiba. Teocomunicação, 43(1), 145-169.

Zaniol, E., Dal Molin, F. \& Andreoli, G. (2007). Um projeto de multiplicidade entre pesquisa, extensão, universidade e comunidade: vivenciando a cultura no bairro Restinga. Revista do Departamento de Psicologia - UFF, 19(1), 227-240. 